\title{
CARACTERÍSTICAS FÍSICOQUÍMICAS Y CALIDAD DEL POZOLE DEL MAÍZ CACAHUACINTLE PROCESADO MEDIANTE TRES MÉTODOS
}

\section{PHYSICOCHEMICAL CHARACTERISTICS AND POZOLE QUALITY OF CACAHUACINTLE MAIZE PROCESSED BY THREE METHODS}

\author{
María Gricelda Vázquez Carrillo ${ }^{\star}$ y David Santiago Ramos
}

Laboratorio de Calidad de Maíz, Campo Experimental Valle de México. Km. 13.5 Carretera Los Reyes-Texcoco, 56250. Coatlinchán, Texcoco, Estado de México. Tel.:(595) 9212657 Ext. 106.

*Autor para correspondencia (gricelda_vazquez@yahoo.com)

\section{RESUMEN}

El pozole es un platillo típico de México, elaborado con granos de maíz (Zea mays L.) suave, nixtamalizado en forma tradicional y hervido hasta que el grano forma una estructura parecida a una flor (grano floreado). En el altiplano se usa el maíz Cacahuacintle para el pozole. En el método comercial se nixtamaliza el grano con cal e hidróxido de sodio, se elimina el pedicelo mecánicamente, se blanquea $(20 \mathrm{~h})$ con metabisulfito de sodio y ácido acético, y finalmente el consumidor lo hierve hasta que se esponja y florea. Un método alternativo consiste en nixtamalizar el maíz con las cantidades óptimas de hidróxido de calcio y de potasio, en blanquear con metabisulfito de potasio durante un tiempo corto, y florear el grano. Los objetivos de esta investigación fueron: 1) Evaluar la calidad del pozole del maíz Cacahuacintle con los métodos tradicional, comercial y alternativo. 2) Determinar el efecto de estos métodos en la composición química del nixtamal, del grano blanqueado y del grano floreado (pozole). El maíz Cacahuacintle estudiado se cultivó en el ciclo primavera-verano 2011, en Nativitas, Estado de México. Las variables estudiadas (físicas, químicas y de procesamiento) se analizaron con un diseño completamente al azar. Los granos de maíz Cacahuacintle fueron muy suaves y de gran tamaño. Con el método alternativo se alcanzó el mayor volumen de floreado y sus rosetas fueron tan luminosas como las del método comercial. El pozole tuvo menos sodio ( $\mathrm{Na}$ ) que el del método comercial; los contenidos de calcio $(\mathrm{Ca})$ y potasio $(\mathrm{K})$ disminuyeron en los tres métodos, respecto al nixtamal y al blanqueado. Con el proceso alternativo se redujo en $76 \%$ el tiempo de procesamiento y se mantuvo mejor la calidad nutricional del pozole, debido a sus mayores contenidos de lisina, triptófano y calcio, y por la reducción de sodio. El nixtamal del método tradicional registró la mayor humedad, pero requirió el mayor tiempo para florear, y tuvo la menor calidad de grano floreado. En los tres métodos y en diferentes etapas del proceso de elaboración del pozole, los contenidos de almidón y cenizas aumentaron, en tanto que la amilosa, la proteína, los lípidos y el triptófano disminuyeron.

Palabras clave: Zea mays, amilosa, aminoácidos, maíz blanqueado, minerales, "pozole".

\section{SUMMARY}

Pozole is a typical Mexican dish made from soft maize (Zea mays $\mathrm{L}$.) grains, nixtamalized in a traditional way and boiled until grains form a flower-like structure (flowered grain). Cacahuacintle maize is used in the Central Plateau of México to make pozole. In the commercial method, maize grain undergoes nixtamalization with lime and sodium hydroxide, then the pedicel is mechanically removed. Afterwards, nixtamal is blanched $(20 \mathrm{~h})$ with sodium metabisulfite and acetic acid. The consumer boils the grains until they become "flowered grains". An alternative method maize grains by nixtamalization with optimal calcium and potassium hydroxide concentrations; a potassium metabisulfite solution is added for a short time for blanching; and finally, the grain is boiled to obtain "flowered grains". This study 1) evaluated "pozole" quality of Cacahuacintle maize following the traditional, commercial and alternative methods; and 2) determined the effect of these processes on the chemical composition of grains in three phases, nixtamal, blanched grain, and "flowered grain" ("pozole"). Cacahuacintle maize used in this study was grown in the 2011 Spring-Summer cycle at Nativitas, State of México. Measured variables (physical, chemical, and process-related) were analyzed in a completely randomized design. Cacahuacintle maize grains were very soft and large. The alternative method produced the highest volume of "flowered grains", and the resulting "pozole" grains were as bright as grains processed with the commercial method. The "pozole" from the alternative method had less sodium $(\mathrm{Na})$ than that from the commercial method. Concurrently, calcium $(\mathrm{Ca})$ and potassium $(\mathrm{K})$ contents diminished with the three methods, compared to nixtamal and to blanched grains. The alternative method reduced processing time by $76 \%$, and its "pozole" had better nutritional quality due to higher lysine, tryptophan, and calcium contents, and sodium reduction. Nixtamal prepared according to the traditional method registered the highest moisture content, but required longer periods for "flowered grains" production, and had the least quality. For the three methods, and at different stages of the "pozole" elaboration process, starch and ash contents increased, while those of amylose, protein, lipids, and tryptophan diminished.

Index words: Zea mays, aminoacids, amylose, blanched grain, minerals, "pozole".

\section{INTRODUCCIÓN}

El pozole, del náhuatl pozolli, "espumoso", de pozol "espuma", o del cahíta posoli "cocer maíz", es un platillo prehispánico, típico y original de México, que consiste en una sopa de granos de maíz, carne, verduras y sal (Jiménez, 2006; Rodríguez, 2010). El principal ingrediente del pozole es el maíz. De las 59 razas nativas descritas por Sánchez et al. (2000), se ha identificado que para la elaboración de este platillo típico se usan al menos 10 razas en diversas regiones del país: Cacahuacintle, Ancho, Elotes Occidentales, 
Tabloncillo, Jala, Bofo, Harinoso de Ocho, Gordo, Blando de Sonora y Bolita (Ortega, 2003).

Estos maíces se caracterizan por el gran tamaño de sus mazorcas (excepto Bolita) y de sus granos, así como por la suavidad de su endospermo. En los estados del centro del país (Puebla, Estado de México, Tlaxcala, Distrito Federal) la raza más utilizada es Cacahuacintle, que se encuentra limitada a altitudes de 2200 a $2800 \mathrm{~m}$. En los Estados de México, Puebla y Tlaxcala, por ser un tipo de maíz con un nicho ecológico muy específico, los productores que se dedican a su cultivo son únicos en su género, lo que les permite tener ante sí un amplio mercado nacional (Bonifacio et al., 2005). Esta raza ha tenido una mayor distribución y diversificación de su comercialización que las demás, debido a que su principal nicho de consumo es el Valle de México, mientras que las otras razas solo se consumen en regiones muy específicas del país o son utilizadas para autoconsumo (Ortega, 2003).

Actualmente se identifican dos procesos para la elaboración del pozole: el tradicional y el comercial. El primero se realiza en aproximadamente $25 \mathrm{~h}$, e involucra la nixtamalización (precocimiento en agua con hidróxido de calcio $\left[\mathrm{Ca}(\mathrm{OH})_{2}\right]$ (cal) $(0.5$ a $2.0 \mathrm{~g} \mathrm{cal} / 100 \mathrm{~g}$ maíz), el reposo $( \pm$ $16 \mathrm{~h}$ ), el "descabezado" (eliminación manual del pedicelo o pico) que se hace "grano por grano", y finalmente el "floreado" (ebullición final del nixtamal en el que éste se hincha y explota hasta adquirir una forma similar a la de una flor) (Rodríguez, 2010). El pozole puede elaborarse con grano entero o con grano descabezado. El grano entero tiene un menor precio pero demanda más tiempo del ama de casa para descabezar el nixtamal (grano cocido). El uso del maíz descabezado representa una reducción considerable de tiempo y de combustible durante el floreado, además de producir un pozole más suave y agradable al paladar respecto al pozole elaborado con el grano sin descabezar.

Hay pequeñas o medianas industrias que elaboran un grano precocido y envasado que venden al público en diferentes comercios, con el cual la etapa final del proceso (floreado) se realiza en 3 ó $4 \mathrm{~h}$. Las principales fases de este proceso comercial son la nixtamalización, el descabezado y el blanqueado. La nixtamalización se hace con una reducida cantidad de cal (0.3 a 0.5 g/100 g de maíz) e hidróxido de sodio en cantidades variables (que dependen de la empresa y de la experiencia del operario), lo que facilita el desprendimiento del pedicelo. Concluido el cocimiento térmico alcalino, el nixtamal se deposita en el equipo "descabezador", el cual consta de un tanque provisto de un eje transversal con paletas que giran para frotar el nixtamal contra la malla (pared) del tanque, de modo que el pedicelo y el pericarpio del nixtamal se desprenden y se separan con ayuda de un flujo continuo de agua. Al retirar el pedicelo, en la base del grano queda una oquedad por la cual penetra el agua libremente, lo que acelera la gelatinización de los almidones del endospermo. Algunas empresas, posterior al descabezado, hacen un proceso denominado precocción que consiste en poner a ebullición el maíz descabezado con acido fosfórico, durante un tiempo corto (Pineda, 2003; Com. pers. ${ }^{1}$ ).

Se ha observado que el grano con mayor blancura es el más apreciado por el consumidor, de modo que la agroindustria prefiere elaborar un producto con estas características. Por ello, una vez descabezado el grano se hace un proceso de blanqueo con una solución de metabisulfito de sodio (3 a $7.5 \mathrm{~g} / 100 \mathrm{~g}$ de maíz), en la que el grano permanece durante un promedio de $20 \mathrm{~h}$.

La falta de información sobre las dosis óptimas y el tipo de aditivos utilizados en la preparación del maíz precocido y sus efectos en la calidad sensorial y nutricional del producto final, así como la creciente demanda de este producto (Hellin y Keleman, 2013), motivaron el estudio de un método alternativo cuyo objetivo fue establecer las cantidades y los tiempos óptimos de los químicos evaluados durante la nixtamalización y el blanqueado del maíz. El método de procesamiento consistió en nixtamalizar el maíz con cantidades óptimas de los hidróxidos de calcio y de potasio; eliminar el reposo tradicional del nixtamal; separar el pedicelo del nixtamal con una descabezadora mecánica; blanquear el nixtamal con la cantidad óptima de metabisulfito de potasio en lugar del de sodio, y usar menos tiempo de reposo que en el método comercial. Finalmente se buscó eliminar el uso de ácido fosfórico y benzoato de sodio.

La sustitución de los productos sódicos tuvo como propósito reducir la concentración de sodio y sus efectos adversos en la salud humana, puesto que cantidades de sodio mayores de $1500 \mathrm{mg} \mathrm{d}^{-1}$ pueden inducir enfermedades como hipertensión arterial en algunas personas, y acumulación grave de líquidos en personas con insuficiencia cardíaca congestiva, cirrosis o nefropatía (ACCF, 2011). Por otra parte, los sulfitos se han empleando para mantener el color original de los alimentos y evitar la decoloración o el oscurecimiento, y para prevenir el crecimiento de microorganismos (FAO, 2013). No obstante, la Comisión del Codex Alimentarius, declaró que estos preservativos deben consumirse en cantidades moderadas, pues pueden provocar reacciones alérgicas y crisis de asma a quienes padecen este trastorno respiratorio (Taylor et al., 1996). En este contexto los objetivos de esta investigación fueron: evaluar la calidad del pozole preparado con maíz Cacahuacintle con tres procesos (tradicional, comercial y un método alternativo), así como determinar el efecto de estos procesos

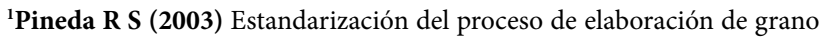
precocido para pozole (Zea mays L.) Tesis profesional. Departamento de Ingeniería Agroindustrial. Universidad Autónoma Chapingo. 
en la composición química del nixtamal, del grano blanqueado y del grano floreado.

\section{MATERIALES Y MÉTODOS}

El maíz Cacahuacintle fue cosechado en el ciclo primavera-verano 2011 en Nativitas, Estado de México, a 1909' $30^{\prime \prime}$ LN y $99^{\circ} 37^{\prime} 17^{\prime \prime}$ LO y una altitud de $2650 \mathrm{~m}$. El clima predominante es templado, con tendencia a frío y subhúmedo. Los suelos son sueltos, muy friables, derivados de cenizas volcánicas y con $\mathrm{pH}$ ácido (entre 4 y 6 ), y descansan sobre un sustrato volcánico de pumicita suelta ("tepojal") con profundidades de 40 a $100 \mathrm{~cm}$.

\section{Características físicas}

En el grano se determinó: peso de 100 granos (PCG), como propusieron Billeb y Bressani (2001); peso hectolítrico (PH), con el método 84-10 (AACC, 2000); índice de flotación (IF), como medida indirecta de la dureza del grano (Vázquez et al., 2011a); y color con el colorímetro Hunter LabMiniScanXE Plus ${ }^{\circledR}$ Modelo 45/0-L (Reston, VA, USA), según la técnica descrita por Vázquez et al. (2012). Las proporciones de pedicelo, pericarpio, germen, endospermo harinoso y endospermo córneo se determinaron mediante la metodología de González (2009). Todas las mediciones se hicieron con dos repeticiones.

\section{Elaboración del pozole}

Se utilizaron tres métodos para la preparación del pozole (grano floreado): el método tradicional, el método comercial, y un tercero denominado "alternativo" (Cuadro 1).

La preparación del pozole por el método tradicional se hizo de acuerdo con la metodología propuesta por Bonifacio et al. (2005).

La elaboración del pozole por el método comercial se basó en la compilación de procesos de dos industrias procesadoras de grano precocido para pozole, los cuales fueron escalados a nivel laboratorio. El proceso consta de cuatro etapas: nixtamalización, descabezado, blanqueado y florea- do. La nixtamalización consistió en colocar 100 g de maíz limpio y de tamaño homogéneo en un vaso de precipitados de $600 \mathrm{~mL}$, agregar agua en relación 2:1 $(200 \mathrm{~mL}$ de agua por $100 \mathrm{~g}$ de maíz) y someter a cocción. Cuando el agua comenzó a hervir se agregaron $0.7 \mathrm{~g}$ de hidróxido de calcio en dosis de $0.7 \mathrm{~g} / 100 \mathrm{~g}$ de maíz, y pasados $2 \mathrm{~min}$ se agregaron $4 \mathrm{~mL}$ de solución de $\mathrm{NaOH}$ a $50 \%(50 \mathrm{~g} \mathrm{NaOH} / 100$ $\mathrm{mL} \mathrm{H}_{2} \mathrm{O}$ ). La nixtamalización duró $60 \mathrm{~min}$, tiempo que se midió a partir de que fue agregado el $\mathrm{NaOH}$.

Una vez concluido el proceso de nixtamalización se descartó el "nejayote" (fase líquida de la cocción) y se lavó el nixtamal con ayuda de una canastilla de plástico y a la vez se frotó el grano contra las paredes para desprender el pedicelo (pico), para así simular el proceso mecánico que se realiza industrialmente con la "descabezadora". Se contaron los granos descabezados y no descabezados. El nixtamal libre de pericarpio y de pedicelo se lavó y se dejó escurrir.

La tercera etapa fue la de blanqueado, en la cual se calentaron $200 \mathrm{~mL}$ de agua a $70^{\circ} \mathrm{C}$, en la que se depositó el maíz descabezado (con temperatura constante de $70^{\circ} \mathrm{C}$ ), se agregaron $6 \mathrm{~g}$ de metabisulfito de sodio $(6 \mathrm{~g} / 100 \mathrm{~g}$ de maíz) y se esperó a que la temperatura bajara a $50{ }^{\circ} \mathrm{C}$. Inmediatamente después se agregaron $2 \mathrm{~mL}$ de ácido acético y se dejó reposar durante $20 \mathrm{~h}$. Al finalizar las $20 \mathrm{~h}$ se sacó el maíz y se descartó la solución de blanqueado. Para la última etapa de floreado, la muestra blanqueada se depositó en un vaso de precipitados con $450 \mathrm{~mL}$ de agua y se sometió a cocción a temperatura de ebullición. El tiempo de floreado se tomó a partir de que la muestra comenzó a ebullir, y se suspendió cuando en una muestra de 10 granos al menos 6 granos estuvieron floreados, momento en que se consideró que al menos $50 \%$ de la muestra cumplía esta característica.

La preparación del pozole por el método alternativo consistió en la nixtamalización del maíz con $1.5 \mathrm{~g}$ de $\mathrm{Ca}(\mathrm{OH})_{2} / 100$ g de maíz, en una solución agua-maíz 2:1, $2.0 \mathrm{~mL}$ de $\mathrm{KOH}$ a $50 \%\left(50 \mathrm{~g} \mathrm{KOH} / 100 \mathrm{~mL} \mathrm{H} \mathrm{H}_{2} \mathrm{O}\right)$ y $60 \mathrm{~min}$ de cocción. El descabezado se hizo de la misma forma que en el método comercial. El blanqueado se hizo con $2 \mathrm{~g}$ de metabisulfito de potasio ( $2 \mathrm{~g} / 100 \mathrm{~g}$ de maíz) y $4 \mathrm{~h}$ de reposo. El floreado se hizo de la misma manera que en el método comercial.

Cuadro 1. Principales diferencias de proceso entre los tres métodos utilizados para la preparación del pozole.

\begin{tabular}{lccc}
\hline \multirow{2}{*}{ Fase } & \multicolumn{3}{c}{ Método } \\
\cline { 2 - 4 } & Tradicional & Comercial & Alternativo \\
\hline Nixtamalización & $\mathrm{Ca}(\mathrm{OH})_{2}$ & $\mathrm{Ca}(\mathrm{OH})_{2}+\mathrm{NaOH}$ & $\mathrm{Ca}(\mathrm{OH})_{2}+\mathrm{KOH}$ \\
Reposo & $16 \mathrm{~h}$ & $\mathrm{NA}$ & $\mathrm{NA}$ \\
Descabezado & Manual & Fricción & Fricción \\
Blanqueado & $\mathrm{NA}^{\dagger}$ & $\mathrm{Na}_{2} \mathrm{~S}_{2} \mathrm{O}_{5}+\mathrm{CH}_{3} \mathrm{COOH}(20 \mathrm{~h})$ & $\mathrm{K}_{2} \mathrm{~S}_{2} \mathrm{O}_{5}(4 \mathrm{~h})$ \\
\hline
\end{tabular}




\section{Calidad del pozole}

Las variables de calidad del pozole fueron: contenido de sólidos en el nejayote, rendimiento, volumen de expansión, humedad, color, dureza, porcentajes de granos descabezados y floreados, tiempo de floreado, y viscosidad del caldo de cocción. La pérdida de sólidos en nejayote, solución de blanqueo y caldo de floreado se obtuvo al cuantificar la materia seca residual después de haber evaporado cada solución y el agua de lavado (Vázquez et al., 2012). El resto de las variables se determinó en nixtamal, grano blanqueado y grano floreado. El rendimiento se expresó como $\mathrm{kg}$ de producto obtenidos por kg de maíz procesado. El volumen de expansión se calculó como la diferencia entre el volumen final y el volumen inicial entre dos etapas del proceso (nixtamal-maíz entero, grano blanqueado-nixtamal), grano floreado-grano blanqueado y grano floreado-nixtamal, según correspondiera el método (Bonifacio et al., 2005).

La humedad se cuantificó por el método 44-10 del AACC (2000); el color se midió con el colorímetro Hunter LabMiniScanXE Plus ${ }^{\circledR}$ Modelo 45/0-L (Reston, VA, USA), como describieron Vázquez et al. (2012), y se registraron las variables $L^{*}$ (luminosidad), ángulo de tono (hue $=\arctan$ $\mathrm{b} / \mathrm{a})$, y pureza de color $\left(\right.$ croma $\left.=\left(\mathrm{a}^{2}+\mathrm{b}^{2}\right)^{1 / 2}\right)$. La dureza se determinó como el pico máximo de fuerza de punción en el perfil de textura obtenido con un texturómetro Brookfield ${ }^{\circledR}$ modelo CT3 (Middleboro, MA, USA) provisto con un puntal de $1.0 \mathrm{~mm}$ de diámetro y $43 \mathrm{~mm}$ de longitud. Los porcentajes de grano descabezado y floreado se calcularon al dividir el número de granos descabezados y floreados entre el total, y multiplicados por 100. La prueba de viscosidad en caldo de cocción se basó en las recomendaciones del manual de operaciones M03-165-E0211 del viscosímetro DV-II+Pro ${ }^{\circledR}$ (Middleboro, MA, USA). La evaluación se realizó a $50 \mathrm{rpm}$ y $25^{\circ} \mathrm{C}$ de temperatura con la aguja SA-02, en una muestra de $200 \mathrm{~mL}$ de caldo puro de cocción del grano de maíz floreado en vasos de precipitados de $250 \mathrm{~mL}$.

\section{Composición química}

Los análisis químicos realizados fueron: contenido de proteína, por el método 46-11 (AACC, 2000); contenido de almidón por el método de Herrera y Huber (1989), de amilosa por el método de Hoover y Ratnayake (2001), de lisina y triptófano con los métodos colorimétricos descritos por Galicia et al. (2009); contenido de lípidos por el método 7.044 (AOAC, 1990) y de cenizas por el método 08-01 (AACC, 2000). Los contenidos de calcio, sodio y potasio se realizaron con la metodología 985.35 de la AOAC (1990).

\section{Análisis estadístico}

Los resultados se obtuvieron por duplicado y se anali- zaron con un diseño completamente al azar. Se hicieron pruebas de comparación de medias (Tukey) y el análisis de correlación simple de Pearson, con el paquete estadístico Statistical Analysis System para Windows, versión 9.0.

\section{RESULTADOS Y DISCUSIÓN}

\section{Características físicas del grano}

El peso hectolítrico (PH) y el índice de flotación (IF) son medidas indirectas de la dureza del grano de maíz, de manera que valores bajos de PH e IF > $87 \%$ son característicos de maíces muy suaves (Vázquez et al., 2003). El maíz Cacahuacintle tuvo un PH de $59.1 \mathrm{~kg} \mathrm{hL}^{-1}$ y un IF de 100 $\%$, un alto contenido de endospermo harinoso (80.6 \%), y nula presencia de endospermo córneo, lo que demuestra la suavidad de los granos de este maíz. El peso de cien granos (PCG) fue de $60.2 \mathrm{~g}$ en promedio, magnitud indicadora de que este maíz tiene granos de tamaño grande (PCG > 38 g) (Salinas et al., 2010), típicos de esta raza. El porcentaje (5.7 \%) y espesor $(108.8 \mu \mathrm{m})$ del pericarpio del maíz Cacahuacintle fue menor al promedio de otros maíces criollos (6.12 \%) (Vázquez et al., 2011a). Estos datos coinciden con los resultados de Narváez et al. (2007), quienes reportaron que los maíces con menor espesor de pericarpio son los más aptos para la elaboración de pozoles. Los altos contenidos de endospermo harinoso y de pedicelo coinciden con los informados por Bonifacio et al. (2005). Por estas características, otro uso especial de este maíz es su consumo como elote (grano inmaduro en etapa lechosa) (Vázquez et al., 2011b).

\section{Calidad del pozole}

La calidad del pozole del maíz Cacahuacintle evaluada por los tres métodos (tradicional, comercial, alternativo) fue expresada por variables de tres fases del proceso de elaboración: nixtamal, grano precocido (blanqueado) y grano floreado (Cuadros 2, 3 y 4). Se encontraron diferencias significativas en todas las variables evaluadas, excepto en rendimiento, humedad y ángulo de tono "hue" en grano blanqueado.

En nixtamal, los mayores valores de rendimiento, volumen de expansión y contenido de humedad se obtuvieron con el método tradicional y los más bajos con el método alternativo. Tanto el rendimiento como el volumen de expansión correlacionaron positivamente con el contenido de humedad $(\mathrm{r}=0.92$ y r $=0.95, \mathrm{P} \leq 0.01$, respectivamente). Esto se explica principalmente porque en el método tradicional el tiempo de reposo $(16 \mathrm{~h})$ permite una mejor hidratación de los gránulos de almidón y un mayor contacto con la solución alcalina, lo que facilita que los iones de calcio penetren en las regiones amorfas ricas en amilosa y se rompan los enlaces intermoleculares y causen 
un alto nivel de hinchamiento (Pineda et al., 2011). Esto resulta en un mayor rendimiento y volumen de expansión.

En los métodos comercial y alternativo, la alta concentración de los hidróxidos de calcio, sodio y potasio, actúa estabilizando los gránulos de almidón y su rigidez, lo que disminuye su volumen. El porcentaje de granos descabezados (GD) en el método tradicional fue de $100 \%$, debido a que fue una actividad manual y se procuró eliminar totalmente el pedicelo. En los otros métodos no se observaron diferencias significativas, aunque el porcentaje de GD fue ligeramente mayor en el método comercial que en el método alternativo, atribuible a que el $\mathrm{NaOH}$ fue más efectivo en la hidrólisis de los compuestos presentes en el pedicelo y eso facilitó retirarlo con mayor facilidad.

El método alternativo produjo el mayor contenido de sólidos $(8.4 \mathrm{~g} / 100 \mathrm{~g})$ en el nejayote y el tradicional el menor ( $4.9 \mathrm{~g} / 100 \mathrm{~g})$, debido a la concentración de reactivos utilizada. Resultados similares fueron obtenidos por Singh et al. (1997), quienes encontraron el mayor grado de efectividad en el descascarillado de maíz con el $\mathrm{NaOH}$, seguido del $\mathrm{KOH}$, y muy por debajo el $\mathrm{Ca}(\mathrm{OH})_{2}$. Durante la nixtamalización los álcalis hidrolizan gran parte del pericarpio, por lo que la única estructura que permanece en el grano es la capa de aleurona, la cual adquiere una tonalidad amarillenta (Bonifacio et al., 2005). El nixtamal lavado fue más luminoso (mayor valor de $\mathrm{L}$ ) en el método tradicional, mientras que en los métodos restantes el color del nixtamal fue más amarillo (menor valor de hue) debido al contacto con los hidróxidos de calcio, sodio y potasio, según el caso.

El alto valor de la dureza del grano nixtamalizado con el método comercial se explica por su bajo contenido de humedad $(\mathrm{r}=-0.75, \mathrm{P} \leq 0.01)$. El método tradicional produjo los granos más suaves debido al mayor tiempo de reposo que permitió una hidratación más completa del grano. En los métodos comercial y alternativo no hubo etapa de reposo del nixtamal, de manera que el interior del grano no fue lo suficientemente hidratado y resultó más duro (Cuadro 2).

En los métodos comercial y alternativo para la producción de maíz precocido, la segunda y última etapa después de la nixtamalización comprende el blanqueo del grano para eliminar el color amarillo-verdoso de la capa de aleurona, para así obtener un color blanco más agradable a los consumidores. El rendimiento de grano precocido es de suma importancia para la industria debido a que entre mayor sea el rendimiento obtenido, mayor será la ganancia. Como se muestra en el Cuadro 3, el método alternativo produjo un rendimiento de grano precocido ligeramente superior al comercial, aunque la diferencia no fue significativa. El método alternativo también dio mayores valores de volumen de expansión y de contenido de humedad que el comercial, comportamiento que no es acorde con el tiempo de blanqueo ya que en el método comercial el maíz permaneció $20 \mathrm{~h}$ en tanto que en el alternativo fueron $4 \mathrm{~h}$; sin embargo, fue claro que aún en esta etapa el mayor uso de hidróxido de calcio en el método alternativo permitió que los gránulos de almidón continuaran su hinchamiento.

El método comercial perdió más sólidos en la solución de blanqueo y sus granos blanqueados fueron más luminosos (valores altos de $\mathrm{L}$ ), debido a las $20 \mathrm{~h}$ de permanencia en la solución de metabisulfito de sodio y acido acético. En el método alternativo se utilizó metabisulfito de potasio y solo $4 \mathrm{~h}$ de reposo (menor tiempo de contacto con la solución) y ésta puede ser una de las razones de la menor blancura del grano precocido. La dureza del grano blanqueado fue mayor en el método alternativo que en el método comercial, diferencia que se atribuye al mayor tiempo de blanqueo que ablandó más el grano en el método comercial.

La etapa final que implica la elaboración de este platillo, es el floreado del grano en el cual las características más importantes son el volumen de expansión del grano y el tiempo de floreado (Cuadro 4). Todas las variables mostraron diferencias significativas entre métodos.

Los métodos de nixtamalización tradicional y comercial proporcionaron los mayores rendimientos de grano floreado, en concordancia con sus mayores contenidos de humedad. Sin embargo, el mayor volumen de expansión lo proporcionó el método alternativo. El volumen de expansión y el contenido de humedad del grano floreado fueron similares a los reportados anteriormente por Vázquez et al. (2011b).

Cuadro 2. Calidad del nixtamal del maíz Cacahuacintle obtenido con tres métodos de elaboración de pozole.

\begin{tabular}{|c|c|c|c|c|c|c|c|c|c|}
\hline \multirow{2}{*}{ Método } & \multirow{2}{*}{ Rendimiento $^{\dagger}$} & \multirow{2}{*}{$\mathrm{VE}^{\dagger \dagger}(\mathrm{mL})$} & \multirow{2}{*}{ GD $(\%)$} & \multirow{2}{*}{$\begin{array}{l}\text { Humedad } \\
(\mathrm{g} / 100 \mathrm{~g})\end{array}$} & \multirow{2}{*}{$\begin{array}{l}\text { Sólidos } \\
\text { (g/100 g) }\end{array}$} & \multicolumn{3}{|c|}{ Color } & \multirow{2}{*}{$\begin{array}{c}\text { Dureza } \\
(\mathrm{N})\end{array}$} \\
\hline & & & & & & Luminosidad & $\mathrm{Hue}^{\text {ss }}$ & Croma $^{\xi}$ & \\
\hline Tradicional & $1.5 \mathrm{a}$ & $58.0 \mathrm{a}$ & $100.0 \mathrm{a}$ & $49.0 \mathrm{a}$ & $4.9 \mathrm{~b}$ & $70.9 \mathrm{a}$ & $90.8 \mathrm{a}$ & $22.8 \mathrm{~b}$ & $2.20 \mathrm{c}$ \\
\hline Comercial & $1.4 \mathrm{~b}$ & $34.3 \mathrm{~b}$ & $93.4 \mathrm{ab}$ & $43.8 \mathrm{~b}$ & $8.1 \mathrm{a}$ & $63.4 \mathrm{~b}$ & $87.5 \mathrm{~b}$ & $39.6 \mathrm{a}$ & $2.85 \mathrm{a}$ \\
\hline Alternativo & $1.3 \mathrm{~b}$ & $26.4 \mathrm{c}$ & $91.0 \mathrm{~b}$ & $41.4 \mathrm{~b}$ & $8.4 \mathrm{a}$ & $69.6 \mathrm{a}$ & $90.3 \mathrm{a}$ & $39.5 \mathrm{a}$ & $2.66 \mathrm{~b}$ \\
\hline
\end{tabular}

${ }^{\dagger}\left(\mathrm{kg}\right.$ de nixtamal descabezado/kg maíz $\left.\mathrm{z}^{-1}\right),{ }^{\dagger \dagger} \mathrm{VE}=$ volumen de expansión; ${ }^{9} \mathrm{GD}=$ granos descabezados; ${ }^{9} \mathrm{Hue}=\arctan (\mathrm{b} / \mathrm{a}) ;{ }^{\xi} \mathrm{Croma}=\left(\mathrm{a}^{2}+\mathrm{b}^{2}\right)^{1 / 2}$. Medias con letras iguales no son estadísticamente diferentes (Tukey, 0.05). 
Cuadro 3. Calidad del grano blanqueado (precocido) de maíz Cacahuacintle obtenido por dos métodos.

\begin{tabular}{|c|c|c|c|c|c|c|c|c|}
\hline \multirow{2}{*}{ Método } & \multirow{2}{*}{ Rendimiento $^{\dagger}$} & \multirow{2}{*}{$\begin{array}{l}\mathrm{VE}^{\dagger \dagger} \\
(\mathrm{mL})\end{array}$} & \multirow{2}{*}{$\begin{array}{l}\text { Humedad } \\
(\mathrm{g} / 100 \mathrm{~g})\end{array}$} & \multirow{2}{*}{$\begin{array}{l}\text { Sólidos } \\
\text { (g/100 g) }\end{array}$} & \multicolumn{3}{|c|}{ Color } & \multirow{2}{*}{$\begin{array}{c}\text { Dureza } \\
(\mathrm{N})\end{array}$} \\
\hline & & & & & Luminosidad & Hue & Cromasg & \\
\hline Comercial & $1.73 \mathrm{a}$ & $47.7 \mathrm{~b}$ & $51.8 \mathrm{a}$ & $5.2 \mathrm{a}$ & $81.11 \mathrm{a}$ & $93.80 \mathrm{a}$ & $17.52 \mathrm{~b}$ & $1.38 \mathrm{~b}$ \\
\hline Alternativo & $1.75 \mathrm{a}$ & $62.4 \mathrm{a}$ & $52.2 \mathrm{a}$ & $2.7 \mathrm{~b}$ & $75.95 \mathrm{~b}$ & $92.19 \mathrm{a}$ & $38.16 \mathrm{a}$ & $2.03 \mathrm{a}$ \\
\hline
\end{tabular}

${ }^{\dagger}\left(\mathrm{kg}\right.$ de grano precocido/kg maíz $\left.{ }^{-1}\right) ;{ }^{\dagger \dagger} \mathrm{VE}=$ volumen de expansión; ${ }^{9} \mathrm{Hue}=\arctan (\mathrm{b} / \mathrm{a}) ;{ }^{99} \mathrm{Croma}=\left(\mathrm{a}^{2}+\mathrm{b}^{2}\right)^{1 / 2}$. En el método tradicional no se realiza proceso de blanqueo. Medias con letras iguales no son estadísticamente diferentes (Tukey, 0.05).

Cuadro 4. Calidad del grano floreado de pozole de maíz Cacahuacintle elaborado por tres métodos.

\begin{tabular}{|c|c|c|c|c|c|c|}
\hline Método & Rendimiento $^{\dagger}$ & $\mathrm{VE}^{\dagger \dagger}(\mathrm{mL})$ & $\mathrm{GF}^{9}(\%)$ & Humedad (g/100 g) & Sólidos (g/100 g) & \\
\hline Tradicional & $2.5 \mathrm{a}$ & $204.0 \mathrm{~b}$ & $79.4 \mathrm{~b}$ & $71.3 \mathrm{a}$ & $1.4 \mathrm{a}$ & \\
\hline Comercial & $2.5 \mathrm{a}$ & $209.7 \mathrm{ab}$ & $90.4 \mathrm{a}$ & $69.0 \mathrm{ab}$ & $1.1 \mathrm{ab}$ & \\
\hline \multirow[t]{3}{*}{ Alternativo } & $2.3 \mathrm{~b}$ & $216.6 \mathrm{a}$ & $93.3 \mathrm{a}$ & $68.3 \mathrm{~b}$ & $1.0 \mathrm{~b}$ & \\
\hline & & Color & & \multirow{2}{*}{ Dureza $(\mathrm{N})$} & \multirow{2}{*}{$\mathrm{TF}^{\phi}(\min )$} & $\mathrm{VC}^{\phi \phi}$ \\
\hline & $L^{99}$ & $\mathrm{Hue}^{\xi}$ & Croma $^{\xi \xi}$ & & & Pa.s \\
\hline Tradicional & $69.0 \mathrm{~b}$ & $92.1 \mathrm{~b}$ & $18.1 \mathrm{a}$ & $0.48 \mathrm{~b}$ & $149 \mathrm{a}$ & $0.019 a$ \\
\hline Comercial & $74.2 \mathrm{ab}$ & $96.8 \mathrm{a}$ & $12.2 \mathrm{~b}$ & $0.46 \mathrm{~b}$ & $114 \mathrm{~b}$ & $0.010 \mathrm{~b}$ \\
\hline Alternativo & $76.3 \mathrm{a}$ & $95.7 \mathrm{a}$ & $14.5 \mathrm{~b}$ & $0.60 \mathrm{a}$ & $103 c$ & $0.011 \mathrm{~b}$ \\
\hline
\end{tabular}

${ }^{\dagger}\left(\mathrm{kg}\right.$ de grano floreado/kg maíz $\left.{ }^{-1}\right) ;{ }^{\dagger \dagger} \mathrm{VE}=$ volumen de expansión; ${ }^{9} \mathrm{GF}=$ granos floreados; $9 \mathrm{~L}=$ luminosidad; ${ }^{\xi} \mathrm{Hue}=\arctan (\mathrm{b} / \mathrm{a}) ;{ }^{\xi \xi} \mathrm{Croma}=\left(\mathrm{a}^{2}+\mathrm{b}^{2}\right)^{1 / 2} ;{ }^{\phi} \mathrm{TF}=$ tiempo de floreado; ${ }^{\phi \phi} \mathrm{VC}=$ viscosidad del caldo de cocción. Medias con letras iguales no son estadísticamente diferentes (Tukey, 0.05).

Pese a la eliminación total del pedicelo, el método tradicional requirió de 46 min más que el método comercial para el floreado, y el menor tiempo lo requirieron los granos obtenidos por el método alternativo. Bonifacio et al. (2005) reportaron que los tiempos de floreado del maíz Cacahuacintle fluctúan entre 120 y 218 min con el método tradicional, y atribuyen un menor tiempo de floreado a la presencia de granos grandes y redondos, mientras que el mayor tiempo de floreado correspondió a granos de forma apastillada triangulada o redondeada.

En el presente estudio los granos fueron redondos-globosos y el tiempo de floreado se asemejó al reportado por esos autores, y fue tres veces menor al reportado por Vázquez et al. (2011b). El porcentaje de granos floreados no se vio favorecido por el descabezado, aunque se esperaba que entre mayor fuese el tiempo de floreado mayor fuera el porcentaje de granos floreados (Bonifacio et al., 2005); sin embargo, el comportamiento observado fue contrario a lo esperado. El color del grano floreado fue más blanco ( $\mathrm{L}^{\star}$ hue y croma) en los métodos comercial y alternativo, lo cual es resultado del proceso de blanqueado al que se sometió el nixtamal antes del floreado, proceso que no se realizó en el método tradicional. El contenido de sólidos en el caldo de cocción fue mayor en el grano floreado por el método tradicional, aunque esto es irrelevante debido a que los compuestos lixiviados se consumen en ese caldo. La pérdida de sólidos y la viscosidad del caldo de cocción correlacionaron positi- vamente entre sí $(\mathrm{r}=0.95, \mathrm{P} \leq 0.01)$, lo cual significa que a mayor pérdida de solidos mayor viscosidad del caldo de cocción, similar a los resultados reportados por Bonifacio et al. (2005).

\section{Composición química del grano entero y pozole}

El contenido de almidón en el nixtamal, expresado en base seca, presentó un aumento con respecto al grano entero, como resultado de la pérdida de otras estructuras del grano, como pericarpio y pedicelo, durante la nixtamalización. Mientras que en el grano blanqueado hubo una ligera disminución, que refleja una pérdida de almidón en la solución de blanqueo, como resultado del tiempo que dura este proceso. En el floreado el contenido de almidón disminuyó a medida que aumentó el tiempo de cocción (Cuadro 5).

El contenido de amilosa en el grano fue de $23.9 \mathrm{~g} / 100$ g. Se observó una clara disminución de este componente al pasar de grano entero a grano floreado; el aumento significativo que se observó en el grano blanqueado se debió a la concentración de componentes tras la eliminación del pericarpio y pedicelo en el proceso de nixtamalización. La amilosa y la amilopectina tienen diferente capacidad de hidratación, puesto que la amilopectina se hidrata más fácilmente que la amilosa (Ansari et al., 2010), por lo que se esperaría que a menor contenido de amilosa, y por tanto mayor de amilopectina, el tiempo de floreado fuera menor. 
No obstante, el contenido de amilosa en el grano floreado correlacionó negativamente con el tiempo de floreado $(\mathrm{r}=$ $-0.93, \mathrm{P} \leq 0.01$ ), lo que significa que a mayor tiempo de floreado habrá menor contenido de amilosa. El mismo comportamiento se presentó con el contenido de proteína $(\mathrm{r}=$ $-0.97, \mathrm{P} \leq 0.01$ ). Aunado a lo anterior, durante el floreado del grano se liberan en el caldo de cocción proteínas solubilizadas, almidón y hasta fragmentos de granos floreados (Bonifacio et al., 2005).

El contenido de lípidos en el grano fue de $4.63 \mathrm{~g} / 100 \mathrm{~g}$, que se redujo más durante la nixtamalización comercial que con la nixtamalización alternativa. Esta disminución se debe a la saponificación de los lípidos por el contacto con los hidróxidos de $\mathrm{Ca}, \mathrm{Na}$ y $\mathrm{K}$ durante la nixtamalización (Martínez et al., 2001). Posteriormente, en el blanqueo se observó un incremento de lípidos debido a la concentración de este componente por la eliminación del pericarpio y el pedicelo en el lavado del nixtamal y descabezado. En el floreado se observó una ligera disminución no significativa (Cuadro 5).

El contenido de cenizas en el grano entero se ubicó en $1.31 \mathrm{~g} / 100 \mathrm{~g}$ en promedio, que con la nixtamalización aumentó debido a la incorporación de minerales por el uso de $\mathrm{Ca}, \mathrm{Na}$ y $\mathrm{K}$ durante el proceso. En el grano blanqueado se observó un aumento respecto al grano de nixtamal, lo cual es el resultado de la incorporación de Na y K utilizados en forma de metabisulfitos durante el proceso de blanqueo. Gran parte de esos minerales se perdieron en el proceso de floreado, aunque permanecen en el caldo de cocción (Cuadro 5).

A diferencia de otros maíces de endospermo normal que son deficientes en los aminoácidos esenciales lisina y triptófano, se ha comprobado que el maíz Cacahuacintle pre- senta niveles superiores de estos aminoácidos (Salinas et al., 2009), por lo que es importante su monitoreo durante el proceso de nixtamalización, ya que su destrucción total es indeseable en el producto final. El contenido de triptófano mostró una disminución progresiva en cada una de las etapas del método tradicional. En el método comercial no se detectó triptófano en el grano blanqueado debido a la cantidad de metabisulfito de sodio presente, compuesto que de acuerdo con Lee y Rogers (1988) acelera la degradación del triptófano en presencia de oxígeno y luz. En el método alternativo se observó una ligera disminución de grano entero a nixtamal y de nixtamal a blanqueado, que luego aumentó en el floreado (Figura 1-A).

El contenido de lisina en el nixtamal elaborado por los métodos tradicional y alternativo se incrementó ligeramente respecto al del grano, mientras que en el comercial disminuyó un poco. Posteriormente en el blanqueo se observó un aumento en el método comercial. Resultados similares fueron obtenidos por Sanderson et al. (1978). En el grano floreado del método comercial se mantuvo prácticamente constante, en tanto que en el alternativo aumentó (Figura 1 A). Estos ligeros aumentos del contenido de lisina en nixtamal, grano blanqueado y grano floreado con respecto al grano entero, se deben a que el proceso de nixtamalización aumenta el contenido de lisina disponible en el maíz porque aumenta la solubilidad de las albúminas y globulinas, proteínas que son ricas en este aminoácido, mientras que disminuye la solubilidad de la fracción de zeína, pobres en este aminoácido (Martínez et al., 2002).

El proceso comercial de elaboración de grano precocido implica el uso de aditivos a base de sodio, por lo que es importante conocer el efecto de cada etapa del proceso sobre estos minerales para identificar su concentración en el producto final. En grano entero el mineral con mayor

Cuadro 5. Composición química del maíz Cacahuacintle (promedio + desviación estándar), en las diferentes etapas de la elaboración del pozole con tres métodos: tradicional, comercial y alternativo.

\begin{tabular}{|c|c|c|c|c|c|c|}
\hline Etapa & Proceso & $\begin{array}{l}\text { Almidón } \\
\text { (g/100 g) }\end{array}$ & $\begin{array}{l}\text { Amilosa } \\
\text { (g/100 g) }\end{array}$ & $\begin{array}{l}\text { Proteína } \\
\text { (g/100 g) }\end{array}$ & $\begin{array}{l}\text { Lípidos } \\
\text { (g/100 g) }\end{array}$ & $\begin{array}{l}\text { Cenizas } \\
(\mathrm{g} / 100 \mathrm{~g})\end{array}$ \\
\hline Grano entero & & $73.0 \pm 1.2$ & $23.9 \pm 0.0$ & $8.3 \pm 0.0$ & $4.63 \pm 0.04$ & $1.31 \pm 0.27$ \\
\hline \multirow{2}{*}{ Nixtamal } & Comercial & $77.7 \pm 0.1$ & $20.0 \pm 0.2$ & $7.6 \pm 0.0$ & $2.91 \pm 0.07$ & $1.44+0.01$ \\
\hline & Alternativo & $79.0 \pm 0.2$ & $20.2 \pm 0.4$ & $7.8 \pm 0.0$ & $3.80 \pm 0.07$ & $1.61+0.01$ \\
\hline Blanqueado & Alternativo & $78.6 \pm 0.3$ & $25.0 \pm 0.2$ & $8.3 \pm 0.0$ & $4.13 \pm 0.02$ & $1.29+0.02$ \\
\hline \multirow[t]{3}{*}{ Floreado } & Tradicional & $76.6 \pm 0.0$ & $16.4 \pm 0.4$ & $7.3 \pm 0.0$ & $4.16 \pm 0.11$ & $1.02+0.01$ \\
\hline & Comercial & $77.2 \pm 0.5$ & $21.6 \pm 0.4$ & $7.8 \pm 0.0$ & $4.07 \pm 0.05$ & $0.64+0.02$ \\
\hline & Alternativo & $79.7 \pm 1.8$ & $20.9 \pm 0.2$ & $7.8 \pm 0.0$ & $3.81 \pm 0.03$ & $0.97+0.01$ \\
\hline
\end{tabular}

Valores informados en muestras libres de agua. 
presencia fue el potasio (Figura 1-B). Durante el proceso de nixtamalización el contenido de $\mathrm{Ca}$ y $\mathrm{Na}$ en el nixtamal aumentó considerablemente, y el de potasio disminuyó en los métodos tradicional y comercial. El aumento en el contenido de $\mathrm{Ca}$ y $\mathrm{Na}$ del grano entero a nixtamal se dio a la par del aumento del contenido de cenizas. Estos resultados coinciden con lo reportado por Bressani (2008), quien afirmó que en la nixtamalización el contenido de $\mathrm{Ca}$ y $\mathrm{Na}$ aumentan y el de $\mathrm{K}$ disminuye.

En la etapa de blanqueado el contenido de Ca y K dismi- nuyó en los dos métodos mientras que el de $\mathrm{Na}$ aumentó, sobre todo en el método comercial, donde la concentración de Na pasó de $61 \mu \mathrm{g} \mathrm{g}^{-1}$ en el grano de maíz a $5638 \mu \mathrm{g} \mathrm{g}^{-1} \mathrm{en}$ el grano blanqueado, lo que demuestra que la cantidad de $\mathrm{Na}_{2} \mathrm{~S}_{2} \mathrm{O}_{5}$ y las $20 \mathrm{~h}$ que duró el proceso permitieron al $\mathrm{Na}$ penetrar el grano blanqueado. En lo que respecta al método alternativo, a pesar del uso de $\mathrm{K}_{2} \mathrm{~S}_{2} \mathrm{O}_{5}$ el contenido de $\mathrm{K}$ disminuyó, lo que indica que el tiempo de blanqueo en este método solo influyó en la decoloración del grano, sin que el K penetrara al grano. Es claro que la disminución del contenido de Ca durante el blanqueado en ambos métodos

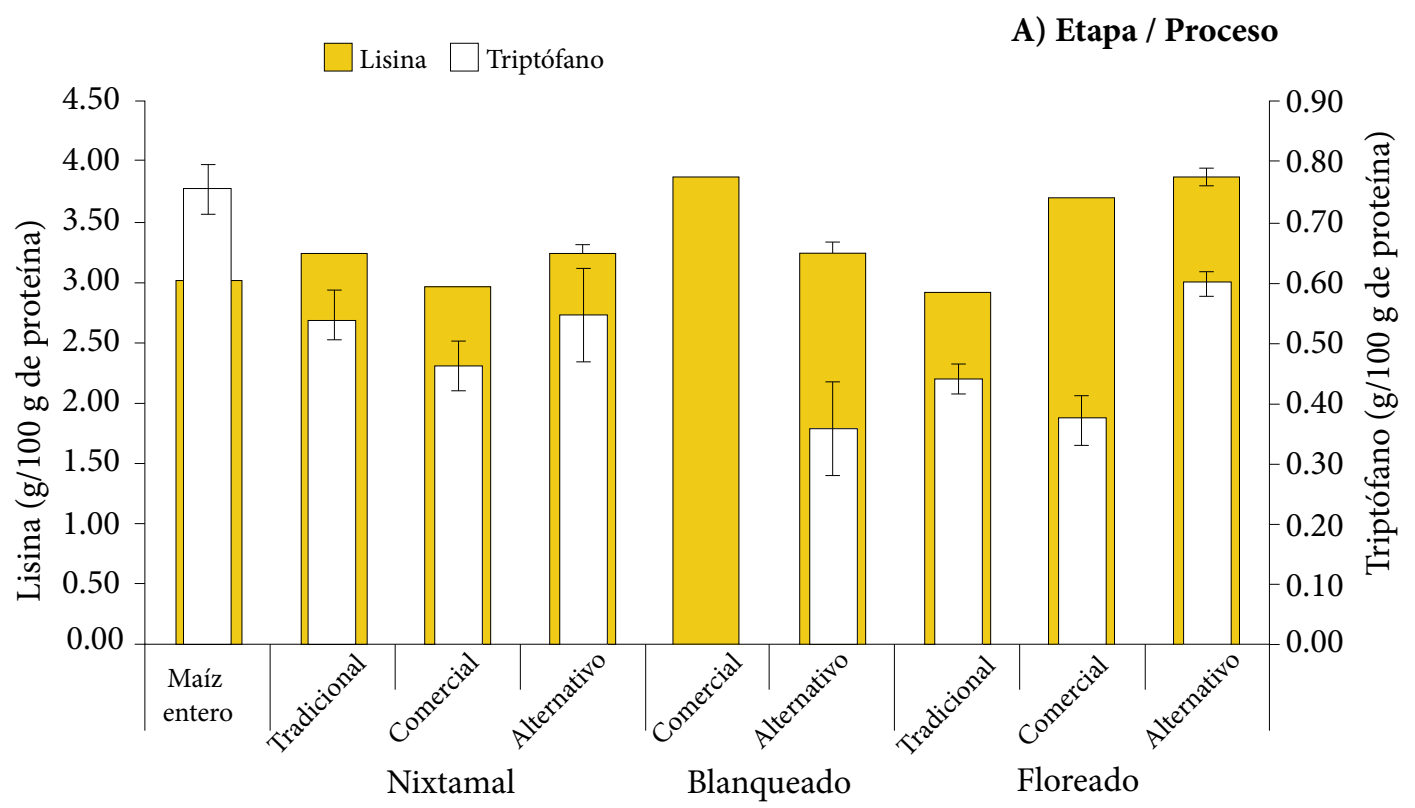

B) Etapa / Proceso



Figura 1. Contenido de lisina y triptófano (A) y de Ca, Na y K (B), en grano de maíz y los productos obtenidos en diferentes etapas de elaboración de pozole, con tres métodos: tradicional, comercial y alternativo. 
se debió a que durante la nixtamalización el calcio solo se adhiere a las capas mas externas del grano sin penetrar al interior (Gutiérrez et al., 2007), además de que no tuvo un tiempo de reposo como en el método tradicional. Durante la etapa de floreado el contenido de todos los minerales disminuyó en los tres métodos, aunque esos contenidos no se perdieron porque permanecieron en el caldo de cocción que finalmente se consume en el pozole.

\section{CONCLUSIONES}

Con el método alternativo se obtuvo la mejor calidad para pozole del maíz Cacahuacintle, debido a que alcanzó el mayor volumen de floreado en menos tiempo, tuvo la menor pérdida de sólidos durante el blanqueado y el floreado del grano, su pozole fue el más luminoso y el de mayor contenido de los aminoácidos esenciales lisina y triptófano. El tiempo de procesamiento se redujo en $76 \%$, respecto al método tradicional. Sus contenidos de calcio y potasio fueron superiores a los del método comercial, en tanto que el sodio se redujo en $43 \%$. Durante la nixtamalización los hidróxidos de calcio y potasio propiciaron mayor pérdida de sólidos, menor rendimiento de grano floreado, siendo éste menos húmedo y más duro.

El método comercial fue el más efectivo para el blanqueado del grano ya que sus granos precocidos fueron los más luminosos y de tono más claro. Su grano floreado requirió menos tiempo para reventar que el del método tradicional; su volumen de floreado, rendimiento y dureza del pozole fueron estadísticamente iguales a los del método tradiciona; además fue el de menor contenido de triptófano. El pozole elaborado con el método tradicional tuvo los mayores valores de humedad, tiempo de floreado, contenido de calcio, potasio, lípidos y cenizas; y registró los menores valores de volumen de floreado, contenido de lisina, almidón, amilosa, proteína, sodio, y de pérdida de sólidos en el nejayote.

\section{BIBLIOGRAFÍA}

AACC, American Association of Cereal Chemists (2000) Approved Methods of the AACC. 16th ed. AACC. The Association. St. Paul, MN. USA. 1200 p.

ACCF, American College of Cardiology Foundation (2011) Expert consensus document on hypertension in the elderly. J. Amer. Coll. Cardiol. 57:1-78.

Ansari O, M Baga, R N Chibbar, N Sultana, N K Howes (2010) Analysis of starch swelling power in Australian breeding lines of hexaploid wheat (Triticum aestivum L.). Field Crops Res. 115:171178.

AOAC, Association of Official Analytical Chemists (1990) Official Methods of Analysis of the AOAC. 15th ed. AOAC, Washington DC, USA. $1230 \mathrm{p}$.

Billeb S A C, R Bressani (2001) Características de cocción por nixtamalización de once variedades de maíz. Arch. Latinoam. Nutr. 51:86-94.

Bonifacio V E I, Y Salinas M, A Ramos R, A Carrillo O (2005) Calidad pozolera en colectas de maíz Cacahuacintle. Rev. Fitotec. Mex.
28:253-260.

Bressani (2008) Cambios nutrimentales en el maíz inducidos por el proceso de nixtamalización: In: Nixtamalización del Maíz a la Tortilla. Aspectos Nutrimentales y Toxicológicos. M E Rodríguez G, S O Serna S, F Sánchez S (eds). Universidad Autónoma de Querétaro. México. pp:19-80.

FAO, Food and Agriculture Organization (2013) Base de Datos en Línea de la Norma General del Codex para los Aditivos Alimentarios (GSFA). Disponible en http://www.codexalimentarius.net/ (Agosto 2013).

Galicia L, E Nurit, A Rosales, N Palacios R (2009) Laboratory Protocols 2009: Maize Nutrition Quality and Plant Tissue Analysis Laboratory. CIMMYT. México. $42 \mathrm{p}$.

González A U (2009) El Maíz y su Conservación. Ed. Trillas. México, D.F. $399 \mathrm{p}$.

Gutiérrez E, I Rojas M, J L Pons H, H Guzmán, B Aguas A, J Arenas, P Fernández, A Palacios F, G Herrera, M E Rodríguez (2007) Study of calcium ion diffusion in nixtamalized quality protein maize as a function of cooking temperature. Cereal Chem. 84:186-194.

Hellin J, Keleman A (2013) Las variedades criollas del maíz, los mercados especializados y las estrategias de vida de los productores. LEISA Rev. Agroecol. 29:7-9.

Herrera S R, J T Huber (1989) Influence of varying protein and starch degradabilities on performance of lactating cows. J. Dairy Sci. 72:1477-1483.

Hoover R, W S Ratnayake (2001) Determination of total amylose content of starch. Curr. Protocols Food Anal.l Chem. E2.3:1-5.

Jiménez M A J (2006) Recuperando significados: El sentido ritual del pozole en la sociedad azteca. Teoría y Praxis 2:25-32.

Lee M G, C M Rogers (1988) Degradation of tryptophan in aqueous solution. PDA J. Pharm. Sci. Tech. 42:20-22

Martínez B F, H E Martínez F, E Sanmartín M, F Sánchez S, Y K Chang, D Barrera A, E Ríos (2001) Effect of the components of maize on the quality of masa and tortillas during the traditional nixtamalization process. J. Sci. Food Agric. 81:1455-1462.

Martínez F H E, F Martínez B, J D Figueroa C, J González H (2002) Studies and biological assays in corn tortillas made from fresh masa prepared by extrusion and nixtamalization processes. J. Food Sci. 67:1196-1199.

Narváez G E D, J D Figueroa C, S Taba (2007) Aspectos microestructurales y posibles usos del maíz de acuerdo con su origen geográfico. Rev. Fitotec. Mex.30:321-325.

Ortega P R (2003) La diversidad del maíz en México: In: Sin Maíz no Hay País. G Esteva, C Marielle (eds). CONACULTA, México pp;123-154.

Pineda G P, D F Coral, D Ramos R, A Rosales R, and M E Rodríguez G (2011) Thermo-alkaline treatment. A process that changes the thermal properties of corn starch. Proc. Food Sci. 1:370-378.

Rodríguez C F (2010) De chile, mole y pozole. Claridades Agropec. 205:43-47.

Salinas M Y, A Ramos R, G Vázquez C (2009) Calidad proteínica en líneas de cuatro poblaciones de maíz Cacahuacintle. In: Mejoramiento, Conservación y Uso de los Maíces Criollos. M B Nájera R, C A Ramírez M (eds). Sociedad Mexicana de Fitogenética. México. pp:48-56.

Salinas M Y, N O Gómez M, J E Cervantes M, M Sierra M, A Palafox C, E Betanzos M, B Coutiño E (2010) Calidad nixtamalera y tortillera en maíces del trópico húmedo y sub-húmedo de México. Rev. Mex. Cienc. Agríc. 1:509-523.

Sánchez G J J, M M Goodman, C W Stuber (2000) Isozymatic and morphological diversity in the races of maize of Mexico. Econ. Bot. 54:43-59.

Sanderson J, J S Wall, G L Donaldson, J F Cavins (1978) Effect of alkaline processing of corn on its amino acids. Cereal Chem. 55:204213.

Singh S K, N Singh, L Du, S R Eckhoff (1997) Effect of sodium hydroxide, calcium hydroxide, and potassium hydroxide on debranning of corn. Cereal Chem 74:254-257.

Taylor S L, R K Bush, J A Nordlee (1996) Sulfites: In: Food Allergy: Adverse Reactions to Food and Food Additives. D D Metcalfe, $\mathrm{H}$ A Sampson, R A Simon (eds). Wiley-Blackwell. USA. pp:339357. 
Vázquez C M G, L Guzmán B, J L Andrés G, F Márquez S, J Castillo M (2003) Calidad de grano y tortillas de maíces criollos y sus retrocruzas. Rev. Fitotec. Mex. 26:231-238.

Vázquez C M G, S García L, Y Salinas M, D J Bergvinson, N Palacios R (2011a) Grain and tortilla quality in landraces and improved maize grown in the highlands of Mexico. Plant Foods Hum. Nutr. 66:203-208.

Vázquez C M G, A Ortega C, M J Guerrero H, B Coutiño E (2011b) Evaluación bioquímica e industrial de razas nativas de maíz de la región serrana de Sonora: In: Amplitud, Mejoramiento, Usos y Riesgos de la Diversidad Genética de Maíz en México. R E Preciado O, F Márquez S (eds). Sociedad Mexicana de Fitogenética. México. pp:97-142.

Vázquez C M G, D Santiago R, Y Salinas M, I Rojas M, J L Arellano V, G A Velázquez C, A Espinosa C (2012) Interacción genotipoambiente del rendimiento y calidad de grano y tortilla de híbridos de maíz en valles altos de Tlaxcala, México. Rev. Fitotec. Mex. 35:229-237. 